\title{
Case Report \\ Pseudotumor Cerebri in the Setting of Differentiation Syndrome in a Patient with Acute Promyelocytic Leukemia Treated with All-trans Retinoic Acid During Induction Therapy
}

Barsha Suwal $M D^{1}$, Bishesh Sharma Poudyal $M D^{2}$, Rabindra Adhikary M.Optometry ${ }^{3}$

${ }^{1}$ Department of Ophthalmology, BP Eye Foundation, Hospital for Children, Eye, ENT and Rehabilitation Services, Bhaktapur, Nepal

${ }^{2}$ Clinical Hematology and Bone Marrow Transplant Unit, Civil Service Hospital, New Baneshwor, Kathmandu, Nepal

3 Tilganga Eye Hospital, Gaushala, Kathmandu, Nepal

1 ORCID id: 0000-0002-1548-3469

2 ORCID id: 0000-0001-5896-0379

3 ORCID id: 0000-0001-5136-1293

\section{Address for correspondence:}

Dr. Barsha Suwal

BP Eye Foundation, Hospital for Children, Eye, ENT and Rehabilitation Services, Bhaktapur, Nepal.

E-mail: suwal.barsha@gmail.com

Phone: +977-9803056313

DOI: https://doi.org/10.3126/

\section{HOW TO CITE}

Suwal B, Poudyal BS, Adhikary R. Pseudotumor Cerebri in the Setting of Differentiation Syndrome in a Patient with Acute Promyelocytic Leukemia Treated with All-trans Retinoic Acid During Induction Therapy. NJNS. 2021;18(4):

To access Nepal Journal of Neuroscience Archives, scan QR code:

Date of submission: $5^{\text {th }}$ August 2021

Date of acceptance: $30^{\text {th }}$ October 2021

Date of publication: $1^{\text {st }}$ December 2021

Running Title: Pseusotumor Cerebri in Differentiation Syndrome

\section{Abstract}




\section{Introduction}

Differentiation syndrome, a well-known complication of all-trans retinoic acid (ATRA) in patients with acute promyelocytic leukemia (APML), can very rarely have ophthalmic manifestations. Pseudotumor cerebri (PC) in the setting of differentiation syndrome (DS) in patients undergoing induction with all-trans retinoic acid has rarely been reported elsewhere. We herein report one such case.

\section{Case Presentation}

A 28-year-old, non-obese female diagnosed as acute promyelocytic leukemia underwent induction with all- trans retinoic acid and Idarubicin. On day 4 of the treatment, she developed high grade fever (104-105 F), dry cough, hypotension, tachycardia, and tinnitus. Chest X ray showed floppy shadows in bilateral lungs. On physical examination, bilateral lower limb edema was noted. She also experienced sudden weight gain of 5 kilogram in 48 hours. After careful exclusion of systemic infection, she was suspected as having DS. She also noticed a reduction in vision in right eye. On eye examination, her best-corrected visual acuity (VA) was 6/60 in the right eye (RE) and 6/6 in the left eye (LE). Fundus evaluation revealed bilateral disc edema with peripapillary hemorrhages along with slight tortuosity of vessels and a yellowish lesion over the fovea in RE. Suspecting DS, she was treated with injection dexamethasone $10 \mathrm{mg}$ twice daily and all- trans retinoic acid was temporarily discontinued. Immediately after its discontinuation, her headache lessened and vision improved gradually. After 2 weeks, her VA was 6/12 in RE and 6/6 in LE which improved to 6/6 in both eyes at 3 months. The patient was also receiving oral voriconazole for fungal prophylaxis and the potentiation effect of all- trans retinoic acid could thus be explained.

\section{Conclusion}

Pseudotumor cerebri associated with all-trans retinoic acid treatment in acute promyelocytic leukemia, even though frequently reported in pediatric patients, is rare in adults. Ophthalmological evaluation is mandatory in all these patients under all-trans retinoic acid therapy.

Key words: ATRA, APML, Case report, Differentiation syndrome, Pseudotumor cerebri.

\section{Introduction}

Acute promyelocytic leukaemia (APML) accounts for roughly $10 \%$ of de novo adult cases of acute myeloid leukaemia (AML-M3) and is characterized by leukemic blast cell morphology, 
coagulopathy and the chromosomal translocation t(15:17). ${ }^{1}$ All-trans retinoic acid (ATRA), a derivative of vitamin $\mathrm{A}$, is a major component of therapy in APML. When combined with anthracycline based chemotherapeutic agents, it yields a complete remission of over $90 \%$ in clinical trials involving APML. ${ }^{1-3}$ However, it has various side effects reported, including differentiation syndrome (DS) and pseudotumor cerebri (PC).

PC in the setting of DS in patients undergoing induction with ATRA has not been reported elsewhere. We herein report one such case.

\section{Case Presentation}

A 28-year- old non-obese, married, lactating female patient presented with a month history of bluish patches over the forearm and thigh for which she sought treatment at a hospital. Complete blood count revealed pancytopenia. Peripheral blood smear showed $67 \%$ abnormal promyelocyte. She was diagnosed with high risk APML, which was later confirmed by morphologic, cytogenetic and molecular analysis of bone marrow.

ATRA $\left(45 \mathrm{mg} / \mathrm{m}^{2}\right.$ in two divided doses) and Idarubicin $(12 \mathrm{mg} / \mathrm{kg}$ for 3 days) were immediately started on the day of presentation. Platelets were transfused daily to keep platelet count above 50,000/ul. Fresh frozen plasma was transfused to keep the fibrinogen level more than 100 and to normalize prolonged prothrombin time and activated prothrombin time. On day 4 of starting the ATRA therapy, she developed high grade fever (104 - $105 \mathrm{~F})$, cough, hypotension, tachycardia and tinnitus. On physical examination, bilateral lower limb edema was noted. She also experienced sudden weight gain of 5 kilogram in 48 hours. Chest X-ray revealed bilateral infiltration. Differentiation syndrome (DS) was diagnosed and she was treated with injection dexamethasone $10 \mathrm{mg}$ twice daily. She also noticed transient blurring of vision in the right eye from the same day. On eye examination, her best- corrected visual acuity (VA) was 6/60 in right eye (RE) and 6/6 in left eye (LE). Both pupils reacted sluggishly to direct and consensual light reflexes. There was no relative afferent pupillary defect. Fundus evaluation revealed bilateral disc edema with peripapillary hemorrhages along with slight tortuosity of vessels and a yellowish lesion over the fovea in RE suggestive of exudation which is shown in figure 1.

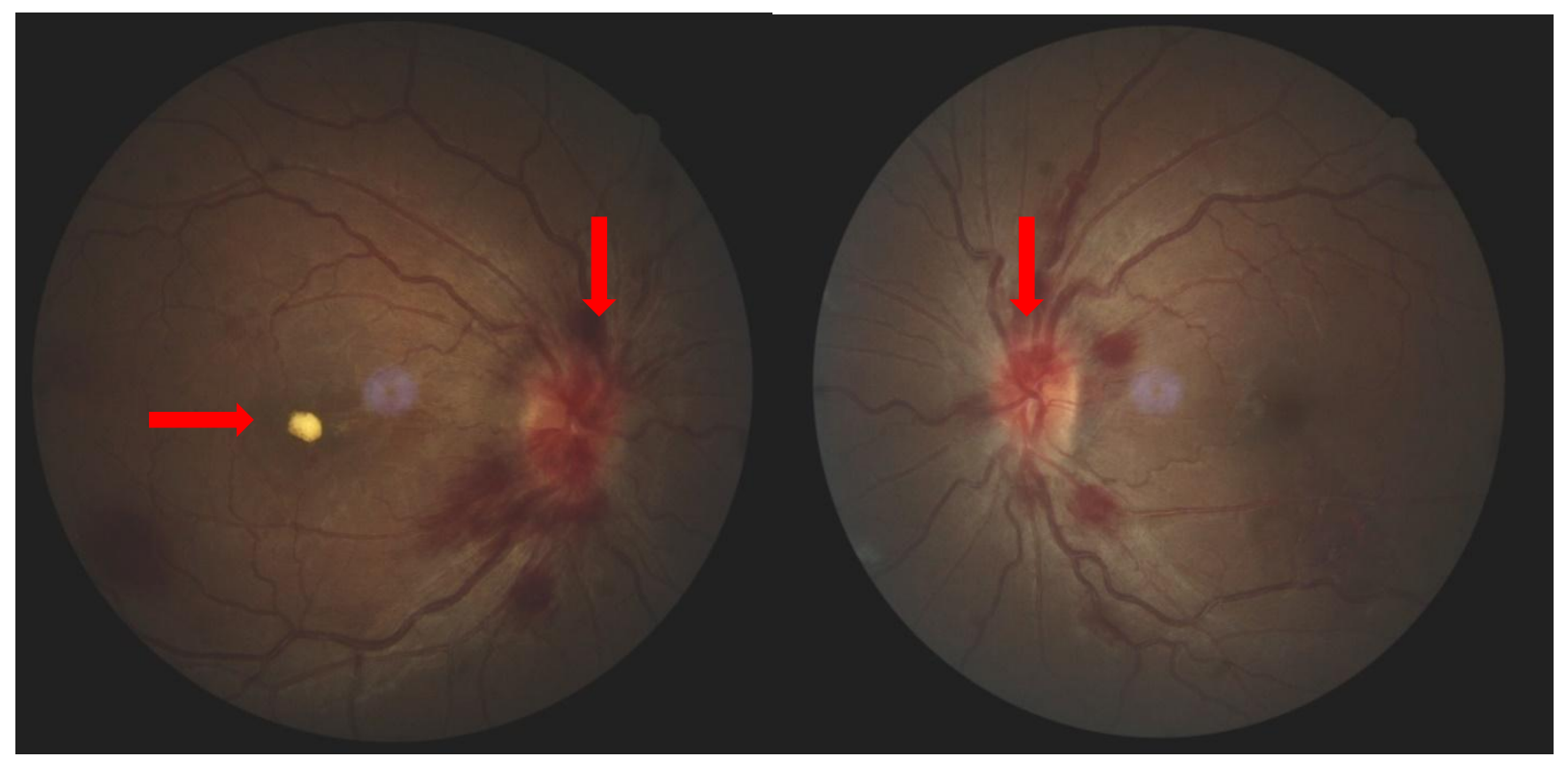


Figure 1: Fundus photographs at the time of presentation show bilateral disc edema with peripapillary hemorrhage in $R E$ and yellowish lesion in right fovea.

Spontaneous venous pulsation of both optic nerve was absent. Color vision with the Ishihara test was normal in both eyes, contrast sensitivity was reduced in RE and normal in LE. Magnetic tomogrance radiography (MRI) head showed no abnormality. Lumbar puncture could not be performed because of a persistent low platelet count. Her other symptoms improved with dexamethasone. However, headache persisted. ATRA induced PC in the setting of DS was sought. As the patient was also on voriconazole and there are reports of triazole antifungals contributing to changes in ATRA concentration, ATRA was stopped and arsenic trioxide was started for the control of APML. Over a period of two days her headache subsided. Vision improved gradually and after 2 weeks, her VA was 6/12 in RE and 6/6 in LE. The disc edema and hemorrhages and yellowish lesion in the fovea had resolved, which is shown in Figure 2 and $3 \mathrm{~B}$ respectively.

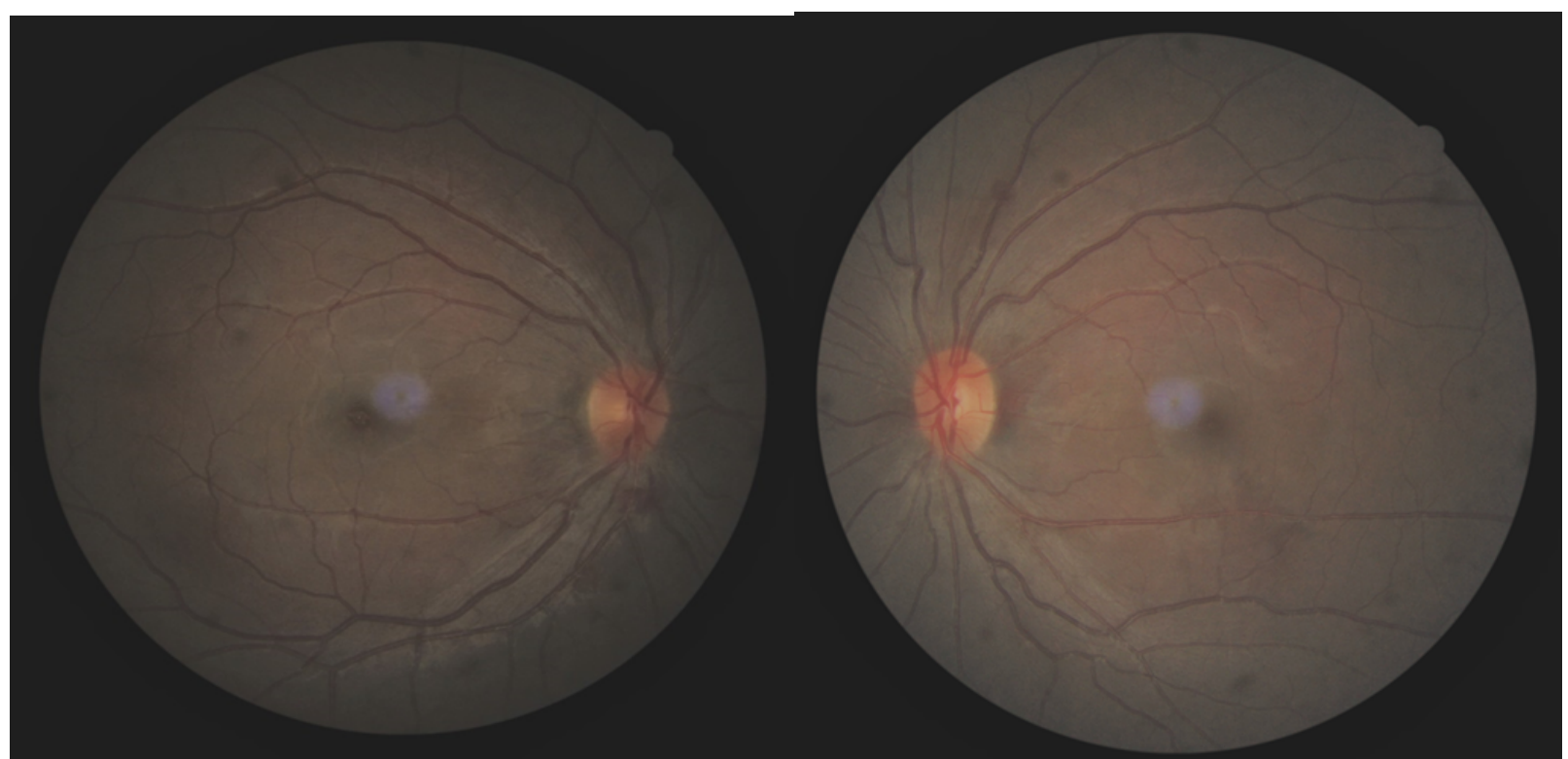

Figure 2: Fundus photographs after 2 weeks of presentation showing resolution of disc edema, tortuosity of blood vessels and yellowish lesion at fovea.

Spectral domain Optical Coherence Tomography (OCT) scan of the RE at initial eye examination showed hyper-reflective lesion in the outer retina with back- shadowing, which is shown in Figure $3 \mathrm{a}$, which resolved later after 2 weeks as shown in Figure 3B. 




Figure 3: OCT scan of the RE at presentation and at 2 weeks following presentation. (A) OCT scan of Right eye showing hyper-reflective lesion in the outer retina over the fovea with backshadowing suggestive of exudation. (B) OCT scan of the same eye which showed a resolved lesion. showing resolution of hyper-reflective lesion in the outer retina.

Automated visual field done at 2 weeks showed blind spot enlargement in RE. At 3 months, her VA was $6 / 6$ in both eyes. Color vision, contrast sensitivity and automated visual field were normal. The patient was given subsequent cycles of chemotherapy, but did not develop reduction in vision, even though she complained of a headache.

\section{Discussion}

In our patient, the visual impairment was coincident with the onset of systemic features of DS with rapid improvement after temporarily stopping ATRA. ATRA, in supra-physiologic doses, is highly efficacious in the treatment of APML. Among other reported side effects of ATRA, less commonly, it has been associated with complications such as DS and PC..$^{4-9}$ The incidence of DS ranges between 2 and $48 \%$ after beginning induction therapy. ${ }^{9-12}$

\section{Differentiation syndrome}

DS, a syndrome typically predominated by unexplained fever and respiratory distress, represents a potentially life-threatening complication of differentiation therapy with reported mortality rates of between $1.0 \%$ and $1.4 \% .^{10-12}$

Ocular manifestations are rarely reported in DS in patients receiving ATRA. In the literature, only three such cases are reported till date, which include chorioretinopathy with multifocal serous neurosensory retinal detachments, choroidal hyperpermeability and bilateral angle closure due to presumed anterior rotation of the iris-lens diaphragm secondary to choroidal thickening. ${ }^{13,14}$ Our patient had bilateral disc edema along with superficial exudation in right eye. The ocular findings and the history was suggestive of PC in the setting of DS.

Pseudotumor cerebri (PC)

Likewise, Pseudotumor cerebri (PC) is a rare but peculiar complication of ATRA therapy, with a reported incidence of $3 \%$ in the largest international trials. ${ }^{3}$ PC associated with ATRA treatment in APML has been frequently reported in pediatric patients, usually within 2 weeks of initiation of treatment. ${ }^{15}$ It is rare in adults as compared to children because of a progressive age-related 
reduction of RAR expression in the central nervous system. ${ }^{16}$ The median time to the diagnosis of PC after beginning of ATRA therapy is 14 days. (Range: 7 days to 10 months). ${ }^{17}$

The pathogenesis of PC secondary to ATRA may be similar to Vitamin A toxicity. One hypothesis suggests that retinoids enhance the production of CSF, disrupt its circulation by altering the lipid constituents of choroid plexus and impair CSF absorption at the level of arachnoid villi or granulations. ${ }^{18}$

\section{Diagnosis of PC}

It is characterized by symptoms and signs of increased intracranial pressure, such as headache, diplopia, and blurring of vision, pulsatile intra- cranial noise. ${ }^{19}$ The diagnosis of PC based on 2013 Modified Dandy criteria includes : (1) presence of papilledema, (2) normal neurologic exam except for cranial nerve abnormalities, (3) normal neuroimaging without evidence of hydrocephalus, mass, or structural lesion, and no abnormal meningeal enhancement on MRI or contrast-enhanced computed tomography, (4) normal cerebral spinal fluid (CSF) composition with elevated lumbar puncture opening pressure.

However, because of the clinical circumstances associated with APML and the frequent inability to perform a lumbar puncture safely in some of these patients, additional criteria was proposed so as to delineate those in whom PC was probable even though they did not meet the full 2013 diagnostic criteria. ${ }^{20}$ Patients with "probable" PTC meet all but one of the diagnostic criteria. "Possible" cases are missing two of the criteria, and "unlikely" cases are those in which an alternate diagnosis was more likely than PTC or when only $0-1$ criteria are present. ${ }^{20}$

In our case, the patient met all the criteria of PC except for lumbar puncture, which was not done. So, she had "probable" pseudotumor cerebri. It is reported that $1.7 \%$ of the patients receiving ATRA, either during induction or maintenance have "probable" PC. ${ }^{20}$

ATRA is oxidized by the cytochrome P-450 system including isoforms CYP2C8, CYP2C9, and CYP3A4. Concomitant administration of drugs that inhibit or are metabolized by this system, most notably triazole antifungals, can lead to toxic ATRA concentrations. ${ }^{21}$ In our case too, the patient was receiving both ATRA and voriconazole, which could explain the potentiation of the side effects of ATRA. Use of oral acetazolamide has been suggested to prevent recurrence.

To conclude, the ocular manifestation during DS may be under-appreciated as visual symptoms in these patients may be un-recognized or deprioritized due to concomitant organ dysfunction. ${ }^{13}$ Hence, a multidisciplinary approach in patients with DS or those receiving differentiation therapy is recommended.

\section{Conclusion}

We herein report PC induced by ATRA in a 28 year old female, which is rare in this age group. To the best of our knowledge, this is the first case reported to have probable pseudotumor cerebri in the setting of differentiation syndrome while undergoing induction therapy with ATRA in a patient with APML. The potentiation effect of ATRA by concomitant use of triazole such as voriconazole is reinforced by our report.

\footnotetext{
Abbreviations:

ATRA: All- trans retinoic acid (ATRA)

APML: Acute promyelocytic leukemia

PC: Pseudotumor cerebri

DS: Differentiation syndrome
} 
VA: Visual acuity

RE: Right eye

LE: Left eye

MRI: Magnetic tomogrance radiography

OCT: Optical Coherence Tomography

\section{Conflict of Interest: None}

Source(s) of support: None

\section{References}

1. Fenaux $\mathrm{P}$, Chastang $\mathrm{C}$, Chevret $\mathrm{S}$ et al. A randomized comparison of all transretinoic acid (ATRA) followed by chemotherapy and ATRA plus chemotherapy and the role of maintenance therapy in newly diagnosed acute promyelocytic leukemia. Blood.1999; 94(4):1192-1200. PMID: 10438706

2. Lo-Coco F, Avvisati G, Vignetti M, et al. Front-line treatment of acute promyelocytic leukemia with AIDA induction followed by risk-adapted consolidation for adults younger than 61 years: results of the AIDA-2000 trial of the GIMEMA Group. Blood. 2010; 116(17): 3171-79. DOI: https://doi.org/10.1182/blood-2010-03-276196

3. Sanz MA, Grimwade D, Tallman MS, et al. Management of acute promyelocytic leukemia: recommendations from an expert panel on behalf of the European LeukemiaNet. Blood. 2009; 113(9): 1875-91. DOI: https://doi.org/10.1182/blood-2008-04-150250

4. Mertelsmann R, Thaler HT, To L. Morphological classification, response to therapy, and survival in 263 adult patients with acute non lymphoblastic leukemia. Blood. 1980; 56(5): 77381. PMID: 6932977

5. Tallman MS, Andersen JW, Schiffer CA et al. All-transretinoic acid in acute promyelocytic leukemia. New England Journal of Medicine. 1997; 337(15): 1021-28. DOI: https://doi.org/10.1056/NEJM199710093371501

6. Simzar S, Rotunda AM, Craft N. Scrotal ulceration as a consequence of all-trans-retinoic acid (ATRA) for the treatment of acute promyelocytic leukemia. J Drugs Dermatol. 2005; 4(2): 23132. PMID: 15776785

7. Jeddi R, Kacem K, Neji HB, et al. Predictive factors of all-trans-retinoic acid related complications during induction therapy for acute promyelocytic leukemia. Hematology. 2008; 13(3): 142-146. https://doi.org/10.1179/102453308X316112

8. Vanier KL, Mattiussi AJ, Johnston D. Interaction of all-trans-retinoic acid with fluconazole in acute promyelocytic leukemia. Journal of Pediatric Hematology/Oncology. 2003; 25(5): 403-404. https://doi.org/10.1097/00043426-200305000-00010

9. Luesink M, Pennings JLA, Wissink WM, et al. Chemokine induction by all-trans retinoic acid and arsenic trioxide in acute promyelocytic leukemia: triggering the differentiation syndrome. Blood. 2009; 114:5512-21. https://doi.org/10.1182/blood-2009-02-204834

10. Leblebjian H, DeAngelo DJ, Skirvin JA, et al. Predictive factors for all-trans retinoic acidrelated differentiation syndrome in patients with acute promyelocytic leukemia. Leuk Res. 2013; 37:747-51. https://doi.org/10.1016/j.leukres.2013.04.011

11. Montesinos P, Bergua JM, Vellenga E, et al. Differentiation syndrome in patients with acute promyelocytic leukemia treated with all-trans retinoic acid and anthracycline chemotherapy: 
characteristics, outcome, and prognostic factors. Blood. 2009; 113:775-783. https://doi.org/10.1182/blood-2008-07-168617

12. Montesinos P, Sanz MA. The differentiation syndrome in patients with acute promyelocytic leukemia: experience of the pethema group and review of the literature. Mediterr J Hematol Infect Dis. 2011; 3(1): e2011059. https://doi.org/10.4084/MJHID.2011.059

13. Levasseur SD, Tantiworawik A. Lambert Maberley DA. All-trans retinoic Acid differentiation syndrome chorioretinopathy: a case of multifocal serous neurosensory detachments in a patient with acute promyelocytic leukemia treated with all-trans retinoic Acid. Retin Cases Brief Rep. 2013; 7: 46-49. DOI: https://doi.org/10.1097/ICB.0b013e3182618d6c

14. Newman AR, Leung B, Richards A, Campbell TG, Wellwood J, Imrie FR. Two cases of differentiation syndrome with ocular manifestation in patients with acute promyelocytic leukemia treated with all-trans-retinoic acid and arsenic trioxide. American Journal of Ophthalmology Case Reports. 2018; 9: 106-11. DOI: https://doi.org/10.1016/j.ajoc.2018.01.026

15. Mahmoud HH, Hurwitz CA, Roberts WM, Santana VM, Ribeiro RC, Krance RA. Tretinoin toxicity in children with acute promyelocytic leukaemia. Lancet. 1993; 342: 1394-5. DOI: https://doi.org/10.1016/0140-6736(93)92755-i

16. Visani G, Bontempo G, Manfroi S, Pazzaglia A, D’Alessandro R, Tura S. All-trans-retinoic acid and pseudotumor cerebri in a young adult with acute promyelocytic leukemia: a possible disease association. Haematologica. 1996; 81(2):152-4. PMID: 8641645

17. Holmes D, Vishnu P, Dorer RK, Aboulafia DM. All-trans retinoic acid-induced pseudotumor cerebri during induction therapy for acute promyelocytic leukemia: A case report and literature review. Case Rep Oncol Med. 2012; 2012:313057. https://doi.org/10.1155/2012/313057

18. Spector RH, Carlisle J. Pseudotumor cerebri caused by a synthetic vitamin A preparation. Neurology. 1984; 34(11): 1509-11. https://doi.org/10.1212/wnl.34.11.1509

19. Friedman DI, Jacobson DM. Diagnostic criteria for idiopathic intracranial hypertension. Neurology. 2002; 59(10): 1492-95. https://doi.org/10.1212/01.wnl.0000029570.69134.1b

20. Coombs CC, DeAngelis LM, Feusner JH, Rowe JM, Tallman MS. Pseudotumor cerebri in acute promyelocytic leukemia patients on Intergroup Protocol 0129: Clinical description and recommendations for new diagnostic criteria. Clin Lymphoma Myeloma Leuk. 2016;16(3):14651. https://doi.org/10.1016/j.clml.2015.11.018

21. Guirgis MF, Lueder GT. Intracranial hypertension secondary to all-trans retinoic acid treatment for leukemia: diagnosis and management. Journal of the American Association for Pediatric Ophthalmology and Strabismus. 2003; 7(6): 432-34. https://doi.org/10.1016/j.jaapos.2003.08.005 